\title{
Retinal Vein
}

National Cancer Institute

\section{Source}

National Cancer Institute. Retinal Vein. NCI Thesaurus. Code C53063.

A vein that drains the retina of the eye. 